\title{
Deformation-Driven Topology-Varying 3D Shape Correspondence
}

\author{
Ibraheem Alhashim $^{1} \quad$ Kai Xu $^{2,3} \quad$ Yixin Zhuang $^{3} \quad$ Junjie Cao $^{4} \quad$ Patricio Simari $^{5} \quad$ Hao Zhang ${ }^{1}$ \\ ${ }^{1}$ Simon Fraser University $\quad{ }^{2}$ Shenzhen VisuCA Key Lab / SIAT \\ ${ }^{3}$ National University of Defense Technology ${ }^{4}$ Dalian University of Technology $\quad{ }^{5}$ The Catholic University of America
}

\begin{abstract}
We present a deformation-driven approach to topology-varying 3D shape correspondence. In this paradigm, the best correspondence between two shapes is the one that results in a minimal-energy, possibly topology-varying, deformation that transforms one shape to conform to the other while respecting the correspondence. Our deformation model, called GeoTopo transform, allows both geometric and topological operations such as part split, duplication, and merging, leading to fine-grained and piecewise continuous correspondence results. The key ingredient of our correspondence scheme is a deformation energy that penalizes geometric distortion, encourages structure preservation, and simultaneously allows topology changes. This is accomplished by connecting shape parts using structural rods, which behave similarly to virtual springs but simultaneously allow the encoding of energies arising from geometric, structural, and topological shape variations. Driven by the combined deformation energy, an optimal shape correspondence is obtained via a pruned beam search. We demonstrate our deformationdriven correspondence scheme on extensive sets of man-made models with rich geometric and topological variation and compare the results to state-of-the-art approaches.
\end{abstract}

CR Categories: I.3.5 [Computer Graphics]: Computational Geometry and Object Modeling-Geometric algorithms, languages, and systems

Keywords: shape correspondence, deformation-driven, topologyvarying, structural rods

\section{Introduction}

Shape correspondence is one of the most fundamental problems in geometry processing [van Kaick et al. 2010]. Earlier approaches have mostly been designed to deal with rigid alignments [Gelfand et al. 2005; Aiger et al. 2008], near-isometric shape articulations [Huang et al. 2008; Kim et al. 2011], or non-rigid shape deformations involving part stretching [Zhang et al. 2008; Wand et al. 2009]. Naturally, with increasing dissimilarity between the shapes being matched, the correspondence search becomes more difficult, both in problem formulation and computational cost.

To handle shapes that differ significantly in geometry and structure, more recent approaches are predominantly knowledge- or datadriven, and resort to supervised learning [van Kaick et al. 2011; Huang et al. 2013] or unsupervised co-analysis [Golovinskiy and
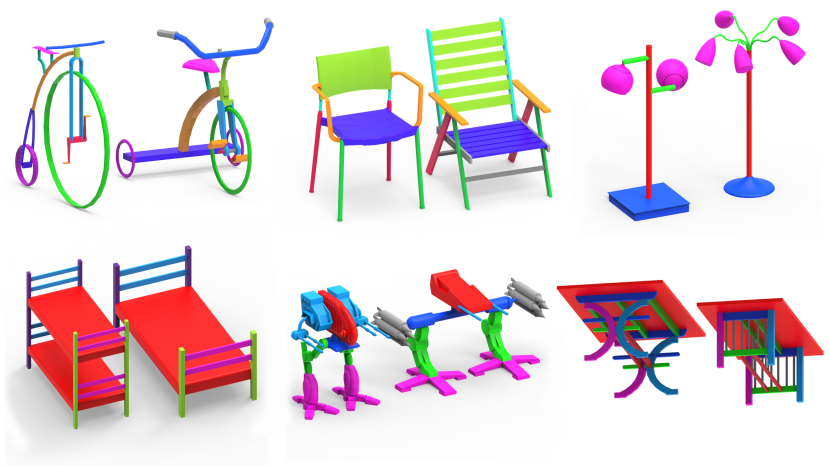

Figure 1: Topology-varying correspondence between man-made shapes exhibiting significant geometry and topology variations. Note the fine-grained and one-to-many matchings between parts.

Funkhouser 2009; Sidi et al. 2011; Xu et al. 2012; Kim et al. 2013; Zheng et al. 2014] of shape collections. By design, these methods only return coarse and discrete correspondences, leaving the structural or topological discrepancies between matched parts or part ensembles unresolved. For example, the wheel assembly of a bicycle is typically matched with that of a tricycle in its entirety.

In this paper, we introduce a novel shape correspondence algorithm which computes a fine-grained mapping between two 3D shapes whose topologies may differ. For example, for the bicycle-tricycle pair above, the fine-level part structures of the two wheel assemblies are matched (see top-left of Figure 1). The fine-grained matching may necessitate part split (e.g., between the front wheel supports in cyan color) and duplication (e.g., between the rear wheels in purple). The resulting correspondence is piecewise continuous. Such a part matching defines a continuous dense correspondence within each pair of matched parts or
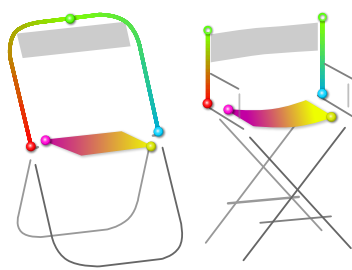
subparts (see the inset).

Our correspondence approach follows a deformation-driven criterion [Sederberg and Greenwood 1992; Blanz and Vetter 1999; Zhang et al. 2008]: the best correspondence between two shapes is the one that incurs a minimum-energy deformation (with an appropriately defined energy) which transforms one shape to conform to the other while respecting the correspondence.

Given two shapes, our correspondence algorithm searches through a space of feasible part correspondences using a pruned beam search [Lowerre 1976], rating them according to the deformationdriven criterion. The key component of our algorithm is a novel deformation model with an associated energy that produces topologyvarying and structure-preserving shape transformations; we call it the GeoTopo transform. The main challenge in developing such a model arises from the many factors that influence its design. Some factors, such as those related to relative part positioning, are dis- 

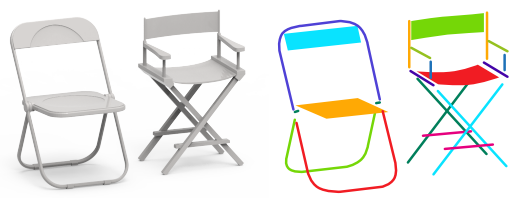

(a) Source (left), target shapes, and curve-sheet abstractions

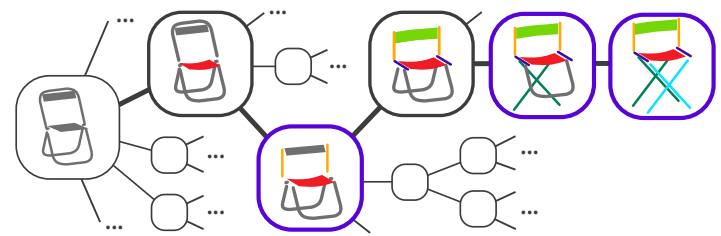

(b) Search tree

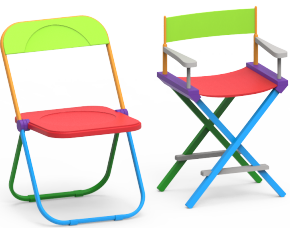

(c) Final correspondence result

Figure 2: An overview of our correspondence scheme. Given a source and a target shape to be matched, we convert each into a skeletal curvesheet representation (a). A combinatorial tree search explores possible one-to-one and one-to-many correspondences between the shape parts (b). Each node in the search tree adds one or more parts to be matched from the source to its parent node. Each assigned correspondence induces a deformation of the source to conform to the target, possibly introducing topological changes (see part splits occurring at nodes marked in purple). The best correspondence from the source to the target (c) is selected from the search path resulting in the least deformation energy. All parts in the source, but not all parts in the target, are matched; unmatched parts are colored in gray.

crete in nature, while others, such as part stretching, are continuous measures. Some factors involve local shape analysis, e.g., part proximity, while others may take a global view of the shapes, e.g., symmetry. The challenge lies in how to unify such a diverse set of factors into a simple and coherent deformation model. The deformation energy needs to penalize geometric distortion, encourage structure preservation, and simultaneously allow topology changes as constrained by the prescribed shape correspondence.

To define the deformation energy, we represent each 3D shape using a structural graph whose nodes are curve and sheet abstractions of the shape parts [Alhashim et al. 2014]. We sample the curves and sheets and connect pairs of sample points between adjacent parts using structural rods. These rods behave like virtual springs in classical physical simulation, but encode energies arising from geometric, structural, and topological shape variations, with more emphasis placed on relative rather than absolute measures. For example, we de-emphasize penalties from stretching or bending of individual parts. Instead, we focus more on structural changes such as connected parts becoming disconnected or angles between two parts changing between two shapes. The energies incurred to deform one shape to another while respecting a prescribed part correspondence between the two shapes are used to rate that shape correspondence during the combinatorial correspondence search.

Our approach is designed to match man-made shapes rich with geometric and topological variation. We show numerous correspondence results over such models, evaluate our method against ground truth data, and compare to prior art, including co-analysis techniques. We also present a key and natural application enabled by the piecewise continuous correspondences obtained: topology-varying shape blending [Alhashim et al. 2014].

\section{Related work}

Shape correspondence, deformation, and structure-aware processing are all well-studied topics with extensive coverage available in recent surveys [van Kaick et al. 2010; Botsch and Sorkine 2008; Mitra et al. 2013]. Most approaches to shape correspondence, including those that are deformation-driven, compute continuous mappings between surfaces to minimize metric distortion. The best known correspondence benchmark [Kim et al. 2011] measures the quality of correspondences using geodesic distortion.

In our work, we compute shape correspondences of a rather different nature. In particular, we allow topological differences between the shapes to be matched and still seek a fine-grained matching between their parts. As such, the matched parts are necessarily split, duplicated, or merged to account for the topological discrepancies. The resulting mapping cannot be continuous; it is, at best, piecewise continuous. In our work, we indeed obtain such mappings through a deformation-driven combinatorial search. This section focuses the discussion to works that are most closely related to our development. Emphasis will be placed on structure-aware deformation models, deformation-driven correspondence, and methods for topological shape processing.

Matching discrete structures. Recent research on shape correspondence is showing an increasing emphasis on dealing with geometric and structural variabilities in the analyzed shapes. Supervised learning is a viable option where correspondence is solved through recognition or semantic labeling [van Kaick et al. 2011]. Unsupervised co-analysis [Mitra et al. 2013] relies on a set, not just a pair, of related shapes to facilitate the discovery of what is common, hence matchable, across the set of shapes.

Recent works on computing discrete part matching between shape pairs address geometric and topological variations by incorporating analysis of part symmetry and contextual relations. The work by Tevs et al. [2014] shows the importance of symmetry in structure matching, resulting in many-to-many correspondences between different shape topologies. However, topological operations are not part of their search and the matching remains at the symmetry group level. Laga et al. [2013] compliment the use of geometric properties with contextual relations between parts when computing shape similarity at a coarse level. Their main goal is to identify shape regions with similar semantics in a supervised manner. Our method is unsupervised and it implicitly encodes contextual relations by considering changes in relative part positioning without the need to explicitly identify and compare such relations.

In contrast to all of these works, which produce discrete and coarse correspondences at the part or part-ensemble level, our work computes fine-grained correspondences, beyond symmetry matching, as well as associated GeoTopo deformations. Our method is unsupervised, works on a pair of shapes only, and is able to deal with significant geometric and topological shape variations.

Deformation-driven correspondence. Early work by Sederberg and Greenwood [1992] takes a deformation-driven approach to compute 2D shape correspondence and blending. Blanz and Vetter [1999] develop a morphable model for correspondence and synthesis of 3D face models. In both cases, the use of shape deformation naturally fits the applications at hand. Huang et al. [2008] perform non-rigid registration of articulated shapes by searching for isometric deformations. The work by Zhang et al. [2008] represents an early attempt at handling significant geometric variation across the shapes being matched. As local feature matching and isometric constraints are no longer applicable, their work searches for a sparse point correspondence that results in a minimal-energy, nonrigid surface deformation. All of these works adopt deformation models for shape boundaries without topology variations. 
Structure-aware deformation. Recent developments on the editing of man-made shapes, led by works on iWires [Gal et al. 2009] and component-wise controllers [Zheng et al. 2011], focus on structure preservation, where shape structures are typically characterized by symmetry and other inter-part relations, such as proximity and co-planarity. Structure-aware retargeting [Bokeloh et al. 2011; Lin et al. 2011] can alter shape topology, but is limited to increasing or decreasing repetition counts in regular or semi-regular patterns. Our work utilizes structure-aware deformation in a correspondence search. The GeoTopo transform models structure preservation similarly to previous works, but the deformation energy must also account for broader topology variations.

Topology matching and blending. There have been works developed to match topological shape characterizations such as Reeb graphs [Hilaga et al. 2001] and curve skeletons [Au et al. 2010], arriving at an estimate of shape similarity and a sparse point correspondence, respectively. In contrast, our method computes a finegrained and piecewise-continuous shape correspondence. More relevant is the recent work by Alhashim et al. [2014], which computes topology-varying blendings between two 3D shapes. They rely on user assistance to establish correspondence between topologically different shapes, which is the task our work attempts to automate. Representation-wise, we adopt the same curve-sheet structural graphs as shape abstractions and a similar set of topologyaltering graph-editing operations. However, we define and rely on a deformation energy to search for the optimal GeoTopo transform, while in their work, the deformations were generated stochastically.

Co-analysis. Unsupervised co-analysis of shape collections has been an intensely studied problem in recent years [Golovinskiy and Funkhouser 2009; Xu et al. 2010; Sidi et al. 2011; Huang et al. 2011; Hu et al. 2012; Xu et al. 2012; Kim et al. 2013; van Kaick et al. 2013; Meng et al. 2013; Zheng et al. 2014]. The essence of co-analysis is to best utilize a set of shapes to infer correspondences across pairs of member shapes. Our work and co-analysis share the common goal of dealing with significant geometric and structural shape variations between the matched shapes. However, in terms of input, co-analysis expects a set of shapes while our work takes a single pair of shapes. In terms of output, co-analysis typically returns a co-segmentation and/or labeling of the shapes that is consistent over the set. Simultaneously addressing a diverse collection in its entirety has meant that a semantically consistent co-segmentation of all member shapes is necessarily coarse. On the other hand, our work aims to return a fine-grained correspondence between a single pair of shapes and their structural differences need not imply a coarsening of the correspondence. Rather, we focus on designing a matching process that allows for such topological differences.

Technique-wise, the co-analysis of Xu et al. [2012] returns a fuzzy part correspondence based primarily on rigid part alignment and Hausdorff distance. The method is applicable both to a pair and a set of shapes, making it a suitable baseline to compare our algorithm. Van Kaick et al. [2013] perform co-hierarchical analysis to obtain one-to-many part correspondences. However, their method does not produce hierarchical matchings with a granularity comparable to that of our algorithm; it also heavily utilizes the entire input set. Perhaps the co-analysis method most relevant to our work is [Kim et al. 2013] due to its use of deforming templates. However, their deformation model does not account for topological variation and, again, the method relies heavily on the entire input set.

\section{Overview}

The input to our algorithm consists of a pair of 3D shapes. We assume that the input shapes are oriented upright and pre-segmented into meaningful parts in a symmetry-aware manner [Wang et al 2011]. We represent both shapes with structural graphs, which encode the shape's topology using medial abstractions of the parts and the structural relations between these abstractions, including symmetry and connectivity. The part correspondence is found by traversing a combinatorial correspondence tree with priority-based pruning where the priority is measured by a self-distortion energy evaluated during deformation of one shape to the other under the constraint of previously matched parts.

The output of our algorithm is a piecewise continuous part-to-part mapping. For example, matched parts abstracted by medial curves would have a continuous mapping between the curves. Figure 2 shows an overview of our correspondence method. To deal with shapes with a large number of parts, we follow a coarse-to-fine strategy when searching for the best correspondence. We enforce correspondence from every part in the source to some part or parts on the target, not vice versa. Thus, multiple source parts could be matched to the same target part, while some target parts might be unmatched. We rely on the deformation energy to decide which source-target designation results in the better solution.

Initial segmentation. Since our method aims to compute finegrained part correspondence, the input shapes are finely segmented. Such segmentations are available from the work of Alhasim et al. [2014], which were obtained manually, and the majority of models appearing in this work come from their dataset. We note that no effort was made to obtain compatible segmentations; each shape was segmented independently.

Structural graph representation. For each 3D shape, we use the shape representation of Alhashim et al. [2014], where segments of the shape represent meaningful parts that are abstracted as medial parametric 1D curves or 2D sheets, extracted via skeletonization, and their interrelations are encoded in a structural graph; see Figure 2(a). While, in their original graph representation, nodes in the graph represent the individual parts, we define each node to represent a symmetry relation (a group of parts) that can be part of a single self-reflectional, -rotational, or -translational symmetry. Each node is labeled with its symmetry group properties and the geometry of a representative part abstraction for the group. Parts colored the same in each shape in Figure 2 (a) visualize the groups we consider in our correspondence search.

Since parts are represented by geometrically simple constructs, the identification of symmetry groups and connectivity is both robust and easy to compute. This is the case especially for the fixed set of supported symmetry groups. Edges of the graph encode connectivity relations between pairs of groups that are in close proximity, connecting a point from each part with minimum distance. We assume that such relations have been computed or provided as part of the input. While the representation of [Alhashim et al. 2014] also encodes a mapping between surface points and the skeletal abstractions, we solely focus on correspondence at the level of abstractions, which can be robust with respect to small topological inconsistencies and large geometric differences.

Self-distortion energy. The key to our correspondence search is a self-distortion energy measured on the source shape. It is formulated to guide the search process to favor and explore correspondence assignments that minimally distort the structure of the original shape. The energy takes into account corresponding groups of parts, which possibly have different cardinalities or abstractions with large geometric differences. We evaluate distortion of the shapes' structure by considering three structural components evaluated before and after the deformation: 1) deviation of spatial arrangement of connected parts, encoded by changes in orientation between sample points on the abstractions; 2) distance between 
parts, encoded by the change in distances between the two closest points across two connected parts; and 3 ) the measure of solidity for parts having their topology altered by a splitting or merging operation induced by a one-to-many part correspondence.

Correspondence search. Since our method allows both one-toone and one-to-many part correspondences, the problem becomes a combinatorial one, for which finding a globally-optimal solution is NP-hard [van Kaick et al. 2010]. We cast this problem as a tree search and solve it using a beam search method augmented with heuristic pruning schemes. The beam search encompasses both global and local pruning. Globally, we keep only the top $K$ optimal solutions for all open search nodes to keep the search space tractable. Locally, for each open search node, we keep only $k$ expansions to ensure an approximately balanced search tree. We further prune based on symmetry groups, requiring all parts within a group to match to some corresponding part or part group.

\section{Energy-driven GeoTopo transform}

Let $M$ and $M^{\prime}$ be the input shapes with corresponding structural graphs $G$ and $G^{\prime}$ and a given (potentially sparse) set of node correspondences between $G$ and $G^{\prime}$. The correspondences may be oneto-one, one-to-many, or many-to-many. In this section, we present the core component of our algorithm: the energy-driven GeoTopo transform, which deforms $M$ to $M^{\prime}$ in an energy-minimizing manner while respecting the given correspondence.

Defining the proper energy to drive the GeoTopo deformation model is key. We first postulate a set of criteria that we believe should influence the design of such a model. The set is fairly substantial and diverse. Some factors are discrete in nature while others are continuous measures. Some factors involve local shape analysis while others take a global view of the shapes. The technical challenge lies in unifying this diverse set of factors into a coherent deformation model. The simplicity of said model and the associated driving energy are an important design choice to achieve efficiency, since the deformation is carried out repeatedly during the combinatorial correspondence search.

\subsection{Factors and motivation}

The GeoTopo deformation model and its energy are designed to simultaneously enforce structure preservation, penalize geometric distortion, and allow topology changes as dictated by the prescribed shape correspondence. In this section, let us describe each of the factors in turn, at a high level.

Structure preservation. We consider terms for enforcing the preservation of symmetry, relative positioning, and connectivity between parts. Symmetries considered include reflectional, rotational, and translational symmetries. With respect to relative positioning, our energy attempts to preserve the spatial relationships between object parts, but not necessarily their exact positioning. Given two parts $A$ and $B$ of the same shape, from a semantic and functional point of view, it is often more important whether $A$ is above, in front, to the left, etc. of $B$, rather than how far above, in front, to the left, etc. $A$ is of $B$. We view these relationships as key aspects of the shape's nature to be preserved in order for it to be remain in its semantic class. Finally, we attempt to preserve all connections between adjacent parts. As we will see, in many cases such constraints must be violated, e.g., when a topology change occurs due to a one-to-many correspondence in parts between the source and target shapes.

Geometric distortion. We consider terms enforcing part distortion and proximity. Part distortion will refer to the degree of defor- mation that a source part will undergo in order to best approximate a part in the target. Parts which were adjacent should remain adjacent if possible, and as proximal as possible otherwise. It should be noted that this term is only applied between parts that are adjacent in the source shape.

Topology changes. We allow for a reduced list of operations to be carried out on the parts of the source shape: split, duplicate, and merge. These operations will allow us to address cases where the number of parts, or their extent, is not in agreement between the source and the target.

The above factors constitute a diverse list of criteria to be taken under consideration. It is very difficult to combine all these factors into a weighted-sum energy function in a principled manner. The required weights for all the terms would yield a high number of parameters that would be difficult to adequately calibrate. This motivates our deformation model, which we present next.

\subsection{Deformation model}

Our approach is to incorporate the factors discussed above into a propagation model. The overarching goal of the deformation model is to respect the given correspondence by moving and deforming parts in shape $M$ to align with the corresponding parts in $M^{\prime}$. As this happens, topology changes may be forced, e.g., when there is a one-to-many correspondence. At the same time, geometric and structural distortions may occur. The unconstrained parts need to be displaced and deformed to minimize the energy resulting from these unavoidable distortions.

First, a correspondence of parts will be determined and these parts will be moved and deformed to fit those of the target shape using the previously-discussed operations. Then, informed by this correspondence, we move the remaining parts via constraint propagation. In this propagation, we wish to preserve structures, including those established by symmetry and connectivity, while simultaneously satisfying constraints given by adjacency. Once a deformed candidate is obtained, we measure the energy of its configuration.

A strong advantage of using the propagation model is that it decouples some of the aspects we wish to consider from the energy formulation, allowing us to keep the latter as light-weight as possible so that it can be effectively used in the correspondence search. The two key properties that are delegated to the propagation are symmetry relations and proximity ("soft" connectivity). We consider these properties to be hard constraints, i.e. shape invariants that, as such, should not be lost during the deformation process. In a sense, these hard constraints can be seen as analogous to mesh connectivity in classical surface deformation. The combined energy and propagation models can thus account for the diverse set of criteria in a compartmentalized and tractable way.

\subsection{Deformation energy}

Existing shape-deformation methods work on satisfying user constraints while minimizing a deformation energy that measures stretching or bending of the shape's initial state. This energy, often modeled with springs, is suitable when the goal is to preserve the overall initial shape of the source as closely as possible. In contrast, we wish to deform the source so that it conforms geometrically to a semantically-related target that is possibly very different in overall shape. Thus, we wish to allow each individual part to undergo large changes in bending and stretching with relatively little penalty.

Similarly to springs, we begin with a system of connected point samples for all pairs of adjacent parts. In traditional mass-spring systems, the energy measures changes in distances between two 

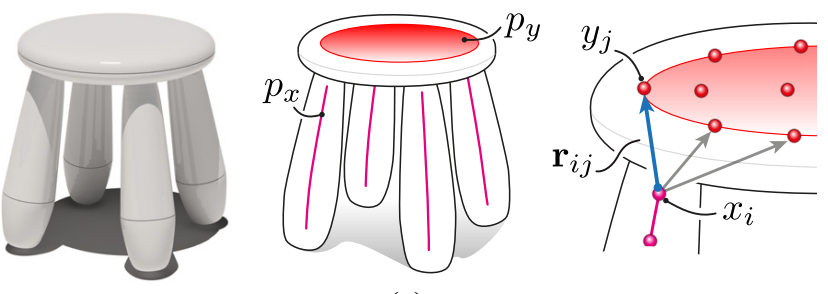

(a)
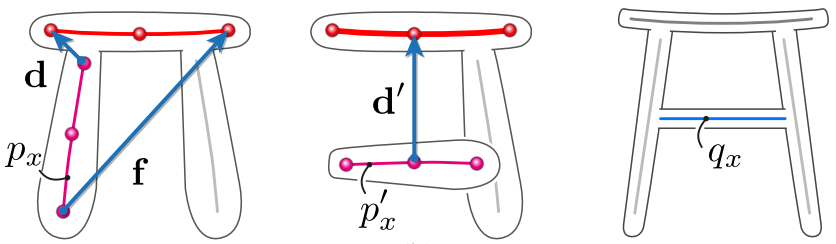

(b)
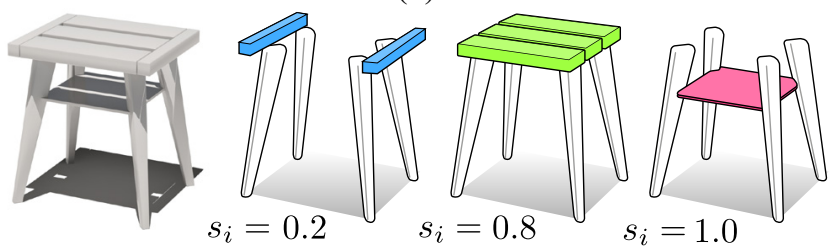

(c)

Figure 3: Deformation energy. For each pair of connected parts, structural rods connect two sample points on the curve-sheet abstractions (only a few rods are shown for clarity) (a). The shortest structural rod is shown before and after ( $d$ and $d^{\prime}$ respectively) a search step for part $p_{x}$ when assigned to part $q_{x}$ on target shape $($ right $)(b)$. In (c) we show the three top choices when searching for correspondence for part $p_{y}$ for the shown target shape (left), and their solidity value s. Note that while the last choice (red) gives the highest $S$ (and thus lowest $E_{s}$ split cost), our total energy favors the middle one (green) as it is lower in both $E_{d}$ and $E_{c}$.

linked points. In contrast, our energy is related to changes in the angle of these connections. These rod-like connections, which we refer to as structural rods, define structural arrangements between parts. A key advantage of this structural-rod paradigm is that it provides a unified treatment of part arrangements and (soft) shape connectivity while minimizing the effect that changes, such as stretching and bending of parts themselves, have on the overall energy.

The key idea of our shape distortion measure is to compute the changes in structural properties resulting from deformation by looking at geometric changes between all pairs of connected parts in the source with an additional penalty function for when topological changes must occur. Conceptually, for each pair of connected parts, we encode an average of bending or twisting on the arrangement of the connected pair before and after a deformation. Our energy consists of three terms:

$$
E=E_{d}+w_{c} E_{c}+w_{s} E_{s}
$$

where $E_{d}$ measures geometric deformation, $E_{c}$ measures pair connectivity, $E_{s}$ is the split penalty for one-to-many and many-to-one part correspondences, and $w_{c}$ and $w_{s}$ are scalar weights.

Part arrangements distortion: The first term, $E_{d}$, captures the geometric changes of two parts $p_{x}$ and $p_{y}$ adjacent in $G$ in terms of their relative spatial arrangement. We begin by uniformly sampling all abstractions (curves and sheets). For each pair of adjacent parts we record a structural rod vector $\mathbf{r}_{i j}$ from the $i$-th sample point on part $p_{x}$ to the $j$-th sample point on the neighboring part $p_{y}, i \in[1, n]$ and $j \in[1, m]$, where $n$ and $m$ are the sample counts of parts $p_{x}$ and $p_{y}$ respectively; see Figure 3(a). The energy of the term is defined by the Frobenius norm of the deformation matrix $D$ comprising all dot products between pairs of pre- and post-deformation structural rods, the latter denoted $\mathbf{r}_{i j}^{\prime}$ :

$$
D_{i j}\left(p_{x}, p_{y}\right)=\max \left(\frac{\mathbf{r}_{i j}}{\left\|\mathbf{r}_{i j}\right\|} \cdot \frac{\mathbf{r}_{i j}^{\prime}}{\left\|\mathbf{r}_{i j}^{\prime}\right\|}, 0\right)
$$

The final normalized form of the deformation energy term is:

$$
E_{d}\left(p_{x}, p_{y}\right)=1-\frac{\|D\|_{F}}{\left\|J_{n m}\right\|_{F}}
$$

where $J_{n m}$ is the unit matrix (one for all elements) of size $n \times m$. Note that the final $E_{d}$ will be the sum of the above term for the entire set of connect pairs on the graph.

Shape connectedness: The second term, $E_{c}$, is the connectivity term, which penalizes disconnection between two originallyadjacent parts by looking at their closest distance. Let $d=$ $\min _{i j}\left\|\mathbf{r}_{i j}\right\|$ and $d^{\prime}=\min _{i j}\left\|\mathbf{r}_{i j}^{\prime}\right\|$ be the length of the shortest pre- and post-deformation structural rods respectively; let $f=$ $\max _{i j}\left\|\mathbf{r}_{i j}\right\|$ be the length of the longest pre-deformation structural rod; see Figure 3(b). If $d^{\prime} \leq d$ (i.e., the parts have actually gotten closer), then we let $E_{c}=0$. Otherwise, we define this term for a pair of adjacent parts as:

$$
E_{c}\left(p_{x}, p_{y}\right)=1-\min \left(1, d^{\prime} / f\right) .
$$

Here too, the final $E_{c}$ will be the sum of the above term for the entire set of connected pairs in the graph.

Topological changes: The final term $E_{s}$ represents the splitting/merging penalty for when such topological changes are needed in the case of one-to-many matches. Since our correspondence works on shapes with differing numbers of parts, it is often the case that many possible matchings can have similarly low energy in both deformation and connection. Figure 3(c) shows one such case. We define a heuristic that takes into account how a split might reflect the apparent intent of a group of similar parts. For example, sparselyplaced bars often function as supports, while a tightly-packed set of bars, or a part abstracted by a sheet, represents a solid part. We define the splitting penalty $E_{s}$ as the deviation in ratio $s$ between the volume of the part to the volume of its convex hull before and after a split (or merge). This shape measure is known as shape solidity in the 2D shape recognition literature where it is defined in terms of areas [Russ 2011]. We encode these ratios for all parts in $G$ in a vector $S$, where $S_{i}=\min \left(s_{i}, s_{i}^{\prime}\right) / \max \left(s_{i}, s_{i}^{\prime}\right)$ for $i \in[1, n], n$ being the number of relations in the source. This penalty does not apply for parts yet to be matched, when matching between single parts, or when matching a rational symmetry group, in which case we set the value of $S_{i}$ to 1 . Similar to Equation 3, we normalize this term as follows:

$$
E_{s}=1-\frac{\|S\|_{F}}{\left\|J_{1 n}\right\|_{F}} .
$$

Each of the three energy terms might individually favor different correspondence choices for the same part (see Figure 4). While, ideally, preservation of the overall structure results in lower values for all terms, a purely geometric approach cannot distinguish between semantically clear but geometrically ambiguous cases. In our energy formulation, we place more value on part arrangements and 


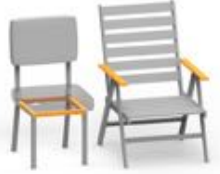

(a) No connection

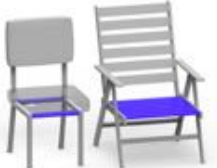

(d) Only deformation

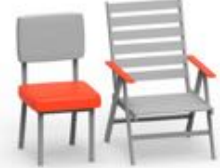

(b) No splitting

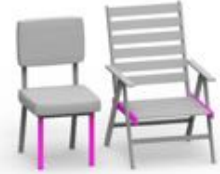

(e) Only splitting

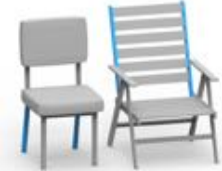

(c) No deformation

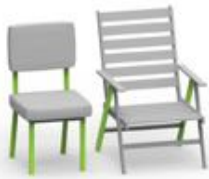

(f) Only connection
Figure 4: Effect of energy terms. Here we highlight obviously bad correspondences between some source and target parts. Connection refers to $E_{c}$, splitting is $E_{s}$, and deformation is $E_{d}$. The entire correspondence computed when enabling all energy terms for this pair of chairs can be found in the gallery.

add the weights $w_{c}$ and $w_{s}$ to balance the contribution of connectivity and topological penalty. We experimentally found the weights $w_{c}=0.6$ and $w_{s}=0.4$ to produce the best results, and we fix these values for all our experiments.

Asymmetry in distortion cost. Input shape pairs, especially ones having different topology, are not expected to share the same connectivity among their corresponding parts. For example, the armrests of chairs might only connect to the seat in one chair while in another they might connect to both the seat and the back. This, in turn, results in an asymmetry of the distortion cost; i.e., deforming $M$ to $M^{\prime}$ may require an energy different from that of deforming $M^{\prime}$ to $M$. In order to symmetrize the distortion cost, one possible heuristic is to take the minimum cost of matching in each direction. However, this heuristic is not guaranteed to always result in the best correspondence and would tend to favor matching the simpler model to the more complex one. This is due to the fact that the simpler model has less elements contributing to the structural distortion. In all of our experiments, we choose the correspondence that incurs the least deformation energy. In practice, this strategy often has the result of leaving extraneous parts of the more complex shape of the input pair unmatched.

Sampling. We sample the parametric curve-sheet part abstractions uniformly at $1 / e$ intervals resulting in curves having $e$ samples and sheets having $e^{2}$ samples. This results in relatively equal contribution for the different parts of the shape. While this sampling might seem counterintuitive, as not all parts of the shape are of equal importance, it ensures that differences between extents of semantically-corresponding parts across different shapes do not significantly bias the results.

\section{Correspondence search}

During correspondence search, we first find correspondences at the group level following a beam search strategy. Then, for a pair of source and target groups in correspondence, we perform partlevel correspondence by first normalizing the bounding boxes of the two groups in order to match individual parts by proximity, and then performing deform-to-fit for the corresponding parts within the source group. Different correspondence choices result in different deformations and thus different energy costs.

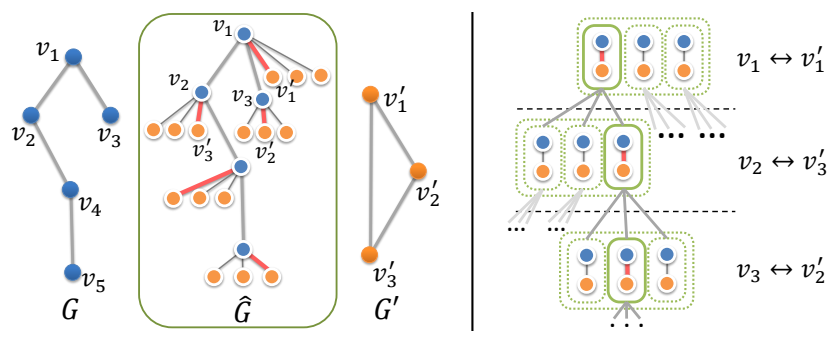

Figure 5: Illustration of matching graph and tree search. Left: The two input structure graphs $G$ and $G^{\prime}$, and their matching graph $\hat{G}$ constructed from $G$. The red edges, together with the original edges from $G$, constitute a solution graph. Right: The search tree constructed while traversing the matching graph in order, where each node corresponds to a matching pair.

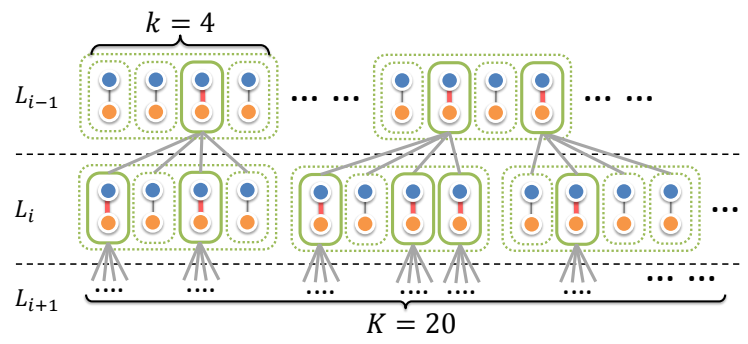

Figure 6: Illustration of the local and global pruning in beam search. When expanding each node with new nodes of matching pairs, we keep only the top $k=4 l$. Among all expanded partial solutions in $L_{i}$, we allow only the top $K=20$ to expand into $L_{i+1}$.

Beam search. Suppose we are given two input shapes, represented by structural graphs $G=\{V, E\}$ and $G^{\prime}=\left\{V^{\prime}, E^{\prime}\right\}$ respectively, where each node in the structural graphs represents a group of parts. Two nodes are connected by an edge if their corresponding surfaces are connected. We construct a super graph $\hat{G}$ from $G$ and $G^{\prime}$ by extending each node $v \in V$ with a set of edges representing matching pairs from $v$ to all nodes in $G^{\prime}$, as shown in Figure 5. A matching in $\hat{G}$ covering all nodes in $G$ represents a full correspondence solution from $G$ to $G^{\prime}$. This is illustrated by the set of red edges in Figure 5. Thus, our problem is reduced to finding such a matching in $\hat{G}$ that minimizes the self-distortion energy; see Equation (1). We employ tree search to solve this problem. The search tree is shown on the right of Figure 5, where each level contains correspondences between a fixed node in $G$ and all other nodes in $G^{\prime}$ and a path from the root to a leaf represents a full correspondence solution between $G$ and $G^{\prime}$ at the group level. Starting from the first node of $\hat{G}$, our algorithm progressively grows the partial solution by traversing the search tree in a branch-and-bound manner. Note that we enforce the correspondence of all groups from the source to match some group(s) on the target. Thus, a target group could be matched from multiple source groups while other target groups might remain unmatched. The correspondence at the part level is resolved in the deform-to-fit step.

To reduce the search space, we introduce two strategies. The first is the use of both local and global pruning during beam search. Locally, when expanding a node in the search tree with different matching nodes, we keep only the $k$ best expansions according to the deformation energy. Globally at each level, among all the newly expanded partial solutions, we retain the top $K$ partial solutions. While the global pruning keeps the overall search space tractable, the local one ensures that the top $K$ partial solutions are 


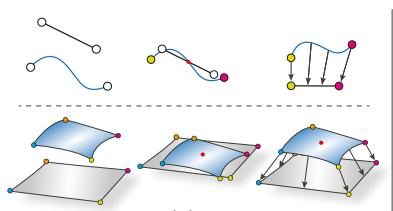

(a)

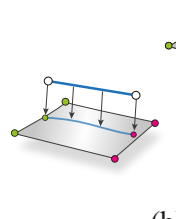

(b)

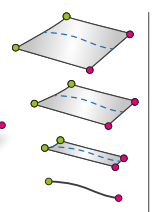

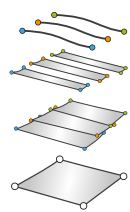

(c)
Figure 7: Illustration of deform-to-fit between curves and sheets. (a): To register a pair of curves(top)/sheets(bottom), we first aligned them at their centers and then find end/corner point matching. (b): To deform a curve into a sheet, we need to project the curve onto the sheet; see the deformation sequence to the right. (c): A deformation sequence from three curves to one sheet, which are also matched by projection.
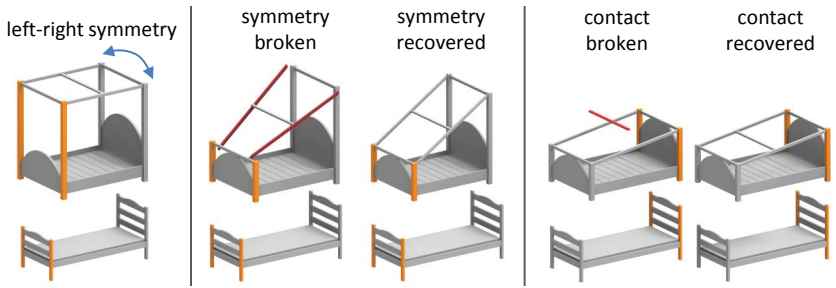

Figure 8: Structure preservation. (a): Two beds with their matched front legs shaded in orange and left-right reflective symmetry marked. $(b)$ : When aligning the matched front legs, the red parts are distorted due to imprecisions in the input (left). Symmetry preservation helps recover the structure (right). (c) After further aligning their back legs, the middle bar in red is detached. Contact constraints are used to recover the connection. Our structure preservation is actually performed on the curve/sheet representation. We show the surfaces for better illustration.

well-distributed among all branches (see Figure 6). This makes our search tree more balanced so that it can cover a wider search space and thus have a higher chance of finding a global optimum. Using lower values of $k$ and $K$ results in faster search times at the risk of missing non-local, closer-to-optimal solutions. We find via experiments that our method is relatively stable to the selection of the two parameters, and $k=4$ and $K=20$ seem to result in a reasonably good trade-off for models containing tens of parts.

The other pruning is based on symmetry groups, requiring parts in the same source group to all match to parts in the corresponding target symmetry group or to a single part that is self-symmetric. In our implementation, we consider only linear-translational, reflectional, and rotational symmetry groups. More aggressive part grouping schemes, such as those based on a symmetry hierarchy [Wang et al. 2011] or recurring part arrangements [Zheng et al. 2014], could also be adopted, but with higher computational cost. Note that the correspondence at the group level is always one-to-one. Required topological changes, including splitting and merging, are decided on during the correspondence search over individual group elements of the source and the target, where the group cardinality may differ.

Deform-to-fit. For each pair of symmetry groups in correspondence, we match their underlying parts by first normalizing the bounding boxes of the two symmetry groups into the unit box and then deforming the parts from the source shape to fit and match against those of the target shape. When each of the two groups contains more than one shape part, we resolve the many-to-many cases into one-to-many instances by looking at the parts' centroids within the unit box and matching them based on proximity. Each source part abstraction, which is a parametric 1D curve or 2D sheet, is deformed to conform to the geometry of its matched part on the target. The deformation of curves or sheets is performed by first aligning their centers, matching their extremities by spatial proximity, and then aligning the extremities by transforming the corresponding end/corner points of the curves/sheets, as shown in Figure 7(a). After aligning the extremities, we linearly interpolate the deformation to the remaining control points.

We also allow parts that are abstracted by curves or sheets to deform into one another. To this end, we first match the extremities between the curve and the sheet. This is achieved by projecting the curve onto the sheet and matching each end of the curve to two corners of the sheet by spatial proximity; see Figure 7(b). This also determines the orthogonal direction in which the curve/sheet will extrude/shrink; see Figure 7(b). To allow groups with differing cardinalities to transform into one another, we need to deal with topological differences; see Figure 7(c). When corresponding one sheet or curve to several curves, we split the sheet or curve part accordingly where appropriate. For one-to-many curve correspondences, we also consider the option of splitting or cutting the source curve into equal-length segments, as shown for the leg and back parts in Figure 2. For all many-to-one cases, we merge multiple parts into a single one as an inverse of the splitting process.

Structure preservation. Deforming parts during the search can result in disconnected shapes, which in turn may break some of the connectivity and/or symmetry relations in the original shape. The evaluation of deformation energy assumes structural plausibility throughout the search, for which we apply an edit propagation step to preserve part relations, similar to prior methods on structureaware shape editing [Gal et al. 2009; Zheng et al. 2011]. In our method, the propagation is constrained by two factors, namely, the connectivity and symmetry relations between parts. For proximity constraints, we preserve the displacement vector between the two connected parts in the source structural graph. This vector is determined by the closest pair of points across the two connected parts. Symmetry is preserved for each symmetry group by simply applying the generation transformation on a single representative element. For reflectional symmetry, this entails a reflection with respect to a plane. For rotational and translational symmetry, transformations are defined with the rotation axis and translation path, respectively. See Figure 8 for a few examples of structure preserving deformation. While our greedy structure-aware deformation better preserves the structural properties of the original shape, there is no guarantee that all constraints are satisfied. More sophisticated constraint satisfactions and their impact on the correspondence performance are worth investigating in future work.

Initial alignment. In order to factor out the large distortions caused by differences in shape scale and rotation, we perform a preprocessing step to roughly align the two shapes to be matched. Specifically, we first scale both models into similar height and then rigidly align them so that their global symmetry planes are parallel. While such an alignment does not resolve symmetric flips, our energy definition would identify such cases by assigning them relatively high distortion costs. In practice, we detect such cases by performing the search with different alignments, choosing the one resulting in the lowest energy cost.

\section{Results, evaluation, and applications}

We show results of topology-varying shape correspondence obtained by our algorithm, evaluate its performance against userspecified ground-truth data, and compare to related correspondence methods, including those designed for co-analysis. We also demonstrate applications that are made possible only when correspon- 

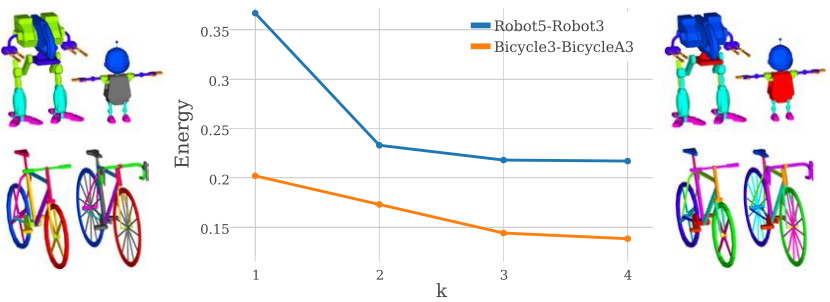

Figure 10: Cost and quality trade-off on two pairs of models. Computational cost is reflected by the beam search width $k$. Quality is defined by the GeoTopo deformation energy.

dences between topologically different shapes are available. All source code and data can be found in the supplementary material.

\subsection{Results and applications}

Input models. In our experiments, we focus exclusively on manmade objects, since all the methods considered are designed to process such objects. We experimented with all shapes (110 of them) from the work of Alhashim et al. [2014] on topology-varying shape blending; these shapes all exhibit rich topological and structural variations. Additional models, including bicycles and tricycles that possess richer variations, were also added into the mix. All the models had been taken from the 3D Warehouse, TurboSquid, and other online sources. Their part counts range between 4 and 35 .

Correspondence results. Figures 1 and 9 show two galleries of shape correspondence results, where we use color matching to visualize the part correspondences with unmatched parts colored gray. Note the significant geometric and structural variations in the matched shapes throughout, as well as the variety of topological changes our algorithm is able to handle, leading to meaningful oneto-many and many-to-many correspondences.

Our algorithm is able to obtain correspondences at a high level of granularity; e.g., see the bicycle-tricycle and table pairs in Figure 1 and the bicycle, boat, cart, and several chair pairs in Figure 9, where fine-scale parts are matched properly.

That being said, it is worth emphasizing that our correspondence scheme is entirely unsupervised. As a result, some of the returned correspondences may not accurately reflect shape semantics or part functionality. One such example is the matching between the legs and leg connections for the fourth pair of chairs in Figure 9.

Statistics and parameters. Table 1 provides timing and other informative statistics for running our algorithm on the model pairs shown in Figure 9. Unless otherwise specified, all correspondence results reported were obtained using the same parameter setting. For controlling the correspondence search, we set $k=4$ and $K=$ 20. For evaluating the correspondences, we fix the weights to $w_{c}=$ 0.6 and $w_{s}=0.4$ in the GeoTopo energy. Note that our method does not require tuning any parameters, making it easily applicable to a set of diverse shape classes.

Computation and quality trade-off. There is an obvious tradeoff between search cost and quality of correspondence results. We provide a simple illustration in Figure 10, where we plot how the deformation energy associated with the final correspondence varies over beam search width $k$, which is a direct indicator for the computational cost of the search. The plots are typical for most model pairs and they allow us to empirically choose a proper set of parameters to obtain a satisfactory trade-off.

\begin{tabular}{rrrrrr}
\hline Pair & $\# P_{s}$ & $\# P_{t}$ & $\# \mathrm{G}_{s}$ & $\# \mathrm{G}_{t}$ & $T(\mathrm{~s})$ \\
\hline Airplane 1 & 6 & 7 & 2 & 3 & 0.06 \\
Airplane 2 & 8 & 29 & 3 & 9 & 0.71 \\
Cart & 13 & 14 & 5 & 5 & 1.09 \\
Table 1 & 7 & 16 & 3 & 7 & 0.32 \\
Weights & 17 & 16 & 8 & 5 & 1.07 \\
\hline Bicycle & 18 & 15 & 4 & 3 & 2.59 \\
Table 2 & 13 & 10 & 3 & 3 & 0.13 \\
Lamp & 12 & 13 & 2 & 1 & 0.19 \\
Bed & 27 & 29 & 5 & 10 & 1.66 \\
Boat & 25 & 17 & 15 & 5 & 2.90 \\
\hline Chair 1 & 12 & 24 & 5 & 8 & 0.96 \\
Chair 2 & 22 & 21 & 8 & 7 & 1.48 \\
Chair 3 & 14 & 12 & 6 & 5 & 0.81 \\
Chair 4 & 12 & 16 & 4 & 4 & 0.92 \\
Chair 5 & 12 & 17 & 7 & 6 & 2.20 \\
\hline
\end{tabular}

Table 1: Statistics and timing for our shape correspondence algorithm, when applied to model pairs shown in Figure 9; the models are listed in left-to-right and top-down order. We report various statistics which influence the search cost: the total number of parts in the source $\left(\# P_{s}\right)$ and target shapes $\left(\# P_{t}\right)$, and the total number of symmetry groups in the source $\left(\# G_{s}\right)$ and target $\left(\# G_{t}\right)$ shapes. Execution times $(T)$, in seconds, account for all correspondence search operations leading to the final result. Timing is measured on an Intel(R) Core (TM) i7, 3.4GHz with 16 GB RAM.

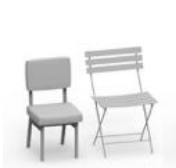

Source and target

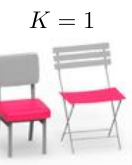

$65 \mathrm{~ms}$

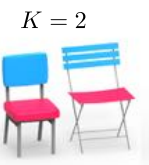

$179 \mathrm{~ms}$

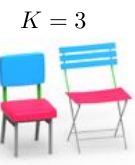

$643 \mathrm{~ms}$

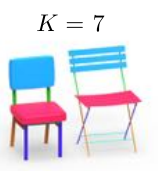

$1371 \mathrm{~ms}$
Figure 11: Coarse correspondences returned by limiting the search to only $K$ parts in the source shape. Here, we consider the $K$ most prominent parts as determined by their volume.

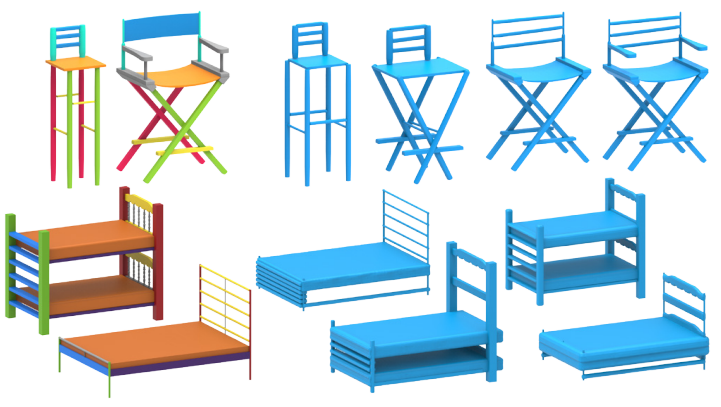

Figure 12: Topology-varying shape blending. Given a source and target shape (left), we show four blending results using part correspondences automatically computed by our method.

Coarse correspondence. While our algorithm can excel at computing fine-grained shape correspondence, it is straightforward to adjust it to return only a coarse set of results. Bounding the part count to $K$ in the final correspondence simply amounts to having the combinatorial search enumerate correspondences with only $K$ parts selected in the source shape. One can also be selective about which $K$ parts to match; e.g., they can be the most prominent ones; see Figure 11. If an application only requires a sparse correspondence, then setting a small $K$ is a viable option for efficiency. With a small $K$, the search can also afford to expand its width, leading to quality improvement in the results. 

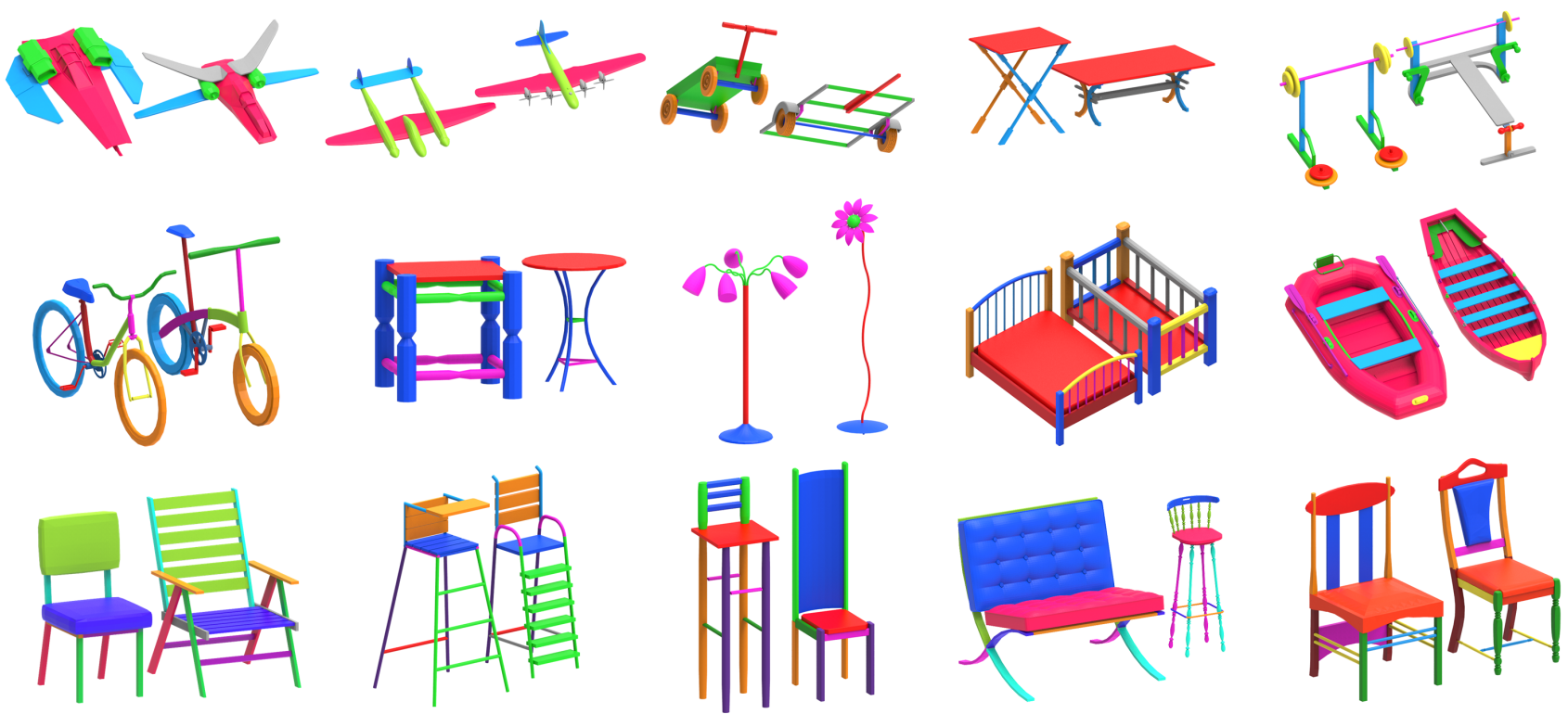

Figure 9: A gallery of fine-grained part correspondence computed by our algorithm. For each pair of input 3D models, we show the best correspondence as rated by the minimal GeoTopo energy. Matched parts share the same color; unmatched parts are in gray.

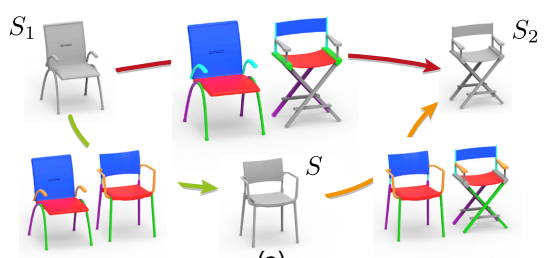

(a)

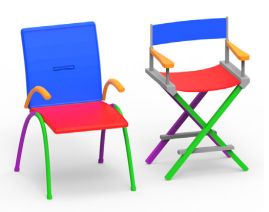

(b)

Figure 13: Transitive shape correspondence. Corresponding the original pairs $S_{1}$ and $S_{2}$ (red arrow) results in improper correspondences with a high energy cost. Whereas computing correspondences by way of $S$ (green and yellow arrows) yields a muchimproved result and a lower sum for the energy cost.

Topology-varying shape blending. A key application enabled by our work is topology-varying blending [Alhashim et al. 2014]. The output of our algorithm is precisely what their tool would require as input. In their work, with significant variation between the source and target shapes, the part correspondences were mostly specified by the user on top of the initial segmentations. Figure 12 shows a few results obtained by their blending tool based on our automatically computed correspondence.

Transitive shape correspondence. Despite our efforts, the correspondence scheme can sometimes fail; e.g., see the result for shapes $S_{1}$ and $S_{2}$ at the top of Figure 13(a). An interesting idea is to have a third shape $S$ that can serve as a bridge or transition between two significantly dissimilar shapes when computing correspondences. By computing correspondences from such a shape $S$ more similar to both shapes (in terms of a proper "correspondence distance") and transitively identifying correspondences from $S_{1}$ to $S_{2}$ through $S$, we obtain an improved result, as shown in Figure 13(b). This idea is most desirable when analyzing a diverse shape collection, among which topology variations are expected. To realize the idea, a topology-varying correspondence scheme is a basic requirement. Our algorithm serves the purpose, with the GeoTopo energy working as a suitable "correspondence distance."
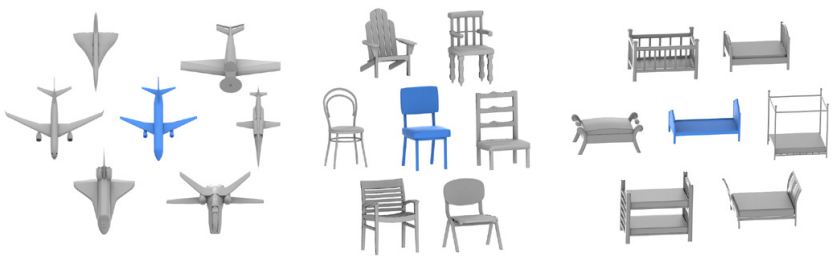

Figure 14: Illustration of topological medoid shapes (blue) computed for different object categories.

"Topological medoids". Related to the use of GeoTopo deformation energy as a correspondence distance between topologically different shapes, we are able to compute a topological medoid for a set of shapes. Give a set of related shapes that differ in their topologies, such a medoid shape is the one in the set having the smallest sum of GeoTopo transformation distances to all other shapes. Specifically, we perform our correspondence algorithm on all shape pairs from the set and assign the deformation energy associated with the final correspondence as the corresponding distance. Similar to a mean shape, the medoid shape can be regarded as a representative shape for the given set. Figure 14 shows topological medoids computed for several small shape collections.

\subsection{Comparison and evaluation}

Ground truth correspondences. In the absence of a benchmark for topology-varying shape correspondence, we have established a dataset for preliminary experiments which consists of 75 shapes covering 5 object categories (chairs, airplanes, tables, beds, and velocipedes) and exhibiting significant geometry and topology variations. Each shape is semantically segmented at a high-level of granularity (e.g., chairs down to individual legs and bicycles down to individual pedals and cross bars). Semantic correspondences between parts within a shape pair or across a set define ground-truth (GT) part correspondences at multiple levels of granularities:

- At the coarse level, the GT correspondences are consistent over the set of 15 shapes for each of the 5 object categories. 

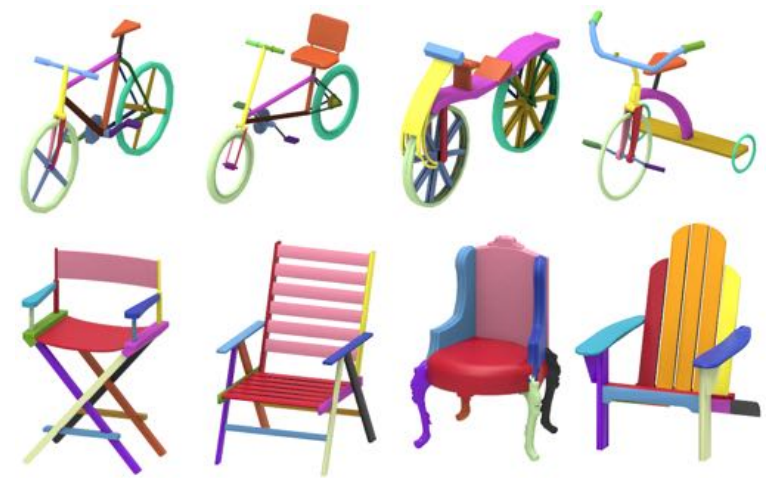

Figure 15: A few shapes and their GT correspondences from our dataset to highlight the challenge the data presents.

For example, the legs of a swivel chair and the four legs of a farm chair would all be labeled as legs. We adopt these correspondences when comparing to co-analysis methods.

- At the finer level, GT correspondences are set up between each pair of shapes belonging to the same category, with the granularity depending on the pair. For example, between a swivel and a four-legged chair, the legs are matched in their entirety, while two four-legged chairs are matched down to "left-front" and "right-back" legs with refined labeling.

Figure 15 shows several shapes with GT correspondences from our dataset, which can well-reflect the difficulty of our test data for the correspondence problem. All shapes and associated multi-level correspondences from the GT dataset are available in the supplementary material, as is the complete source code for our method.

Correspondence measure by precision+recall. Given a pair of shapes with a GT correspondence set (of part pairs) $G$, we would like to assess the quality of a correspondence set $M$ returned by a particular matching scheme. An obvious measure would be the percentage of part pairs in $M$ which belong to $G$ [Zheng et al. 2014]. However, if we make an analogy to retrieval, and regard $G$ as the set of relevant instances and $M$ as the set of retrieved instances, then such a measure would only be accounting for precision, not recall. For example, the fact that some GT correspondences are not found in $M$ would be overlooked. Let $R=M \bigcap G$. Then, we measure both precision $|R| /|M|$ and recall $|R| /|G|$ for a computed correspondence $M$. When making comparisons, we report both precision and recall rates averaged over sets of shape pairs.

Comparison for co-analysis. To compare our method, which is designed to match a shape pair, to co-analysis, we need to adapt our method to handle a shape set. Given a set of shapes, we reconstruct a set (part) correspondence out of all the pairwise correspondences obtained by GeoTopo. This is achieved by encoding the fine-grained part correspondence between all pairs of shapes into a large correspondence matrix and then extracting from the matrix a sparse correspondence via graph clustering [van Dongen 2000]. Each cluster represents a set of parts which are strongly connected by the correspondence and possess a consistent correspondence. This scheme is admittedly preliminary, but it bears some similarity to the correspondence space analysis of Lipman et al. [2010]. Note that we do not utilize any priors over the set nor impose any additional constraints to improve the consistency of the correspondence extracted [Huang and Guibas 2013].

The co-analysis methods we compare to include fuzzy part correspondence [Xu et al. 2012], recurring part arrangements [Zheng

\begin{tabular}{lllll}
\hline Category & {$[\mathrm{Xu} 2012]$} & {$[$ Zheng 14] } & {$[$ Kim 13] } & Ours \\
\hline chair & 0.72 & 0.67 & 0.74 & $\mathbf{0 . 8 3}$ \\
table & 0.62 & 0.35 & 0.32 & $\mathbf{0 . 6 9}$ \\
bed & 0.49 & 0.46 & 0.57 & $\mathbf{0 . 6 1}$ \\
airplane & 0.61 & 0.38 & 0.73 & $\mathbf{0 . 8 4}$ \\
velocipede & 0.66 & 0.37 & 0.56 & $\mathbf{0 . 7 9}$ \\
\hline
\end{tabular}

Table 2: Comparison on per-category precision rates among coanalysis methods. The best rates are shown in bold.

et al. 2014], and perhaps the most closely-related method by Kim et al. [2013] via template fitting. Like GeoTopo, the performance of Zheng et al. [2014] depends on the initial shape graph with symmetry and contact information encoded. We follow descriptions in the paper for filtering out small components based on thresholding and grouping parts based on symmetry relations. We find the best set of parameters to produce finer (consistent) correspondences. However, by design, their output remains coarse compared to others. For the template fitting method, we manually create one template for each tested object category and train the templates over our GT datasets. All three methods were otherwise executed using the optimal parameters as suggested by the respective papers.

Table 2 reports comparison results on precision with respect to the coarse GT. In terms of execution times, Kim et al. [2013] and our method are comparable, while the other two are much faster, with $\mathrm{Xu}$ et al. [2012] being by far the fastest. The results demonstrate superior performance by our method on all object categories tested. For Kim et al. [2013], the performance on the table set is less than satisfactory since some of the tables have crossed legs and some have straight legs. Hence, two templates would have been more suitable. For the velocipede set, many parts intersect each other, which is considered a difficult scenario for the method.

The adaptation of our method for co-analysis is by no means optimal or even sophisticated. Co-analysis is not the focus of this paper; the scheme is designed to allow for a meaningful comparison. However, the superior performance shown suggests the potential of our fine-grained pairwise correspondence scheme to support more accurate co-analysis, in particular, for highly diverse shape collections with topology variations.

Comparison for matching shape pairs. We take the method of $\mathrm{Xu}$ et al. [2012] as a baseline for comparing pairwise shape correspondences. This method is based on global shape alignment and proximity-based part matching, and it is applicable to shape pairs. Based on our experiments, the other co-analysis methods do not perform nearly as well when the input consists not of a set, but rather only two shapes. Table 3 shows a comparison between this baseline and our correspondence method on precision and recall measured against the GT correspondences for all five categories. Our method again appears to outperform Xu et al. [2012] on this test. Note however that the precision within some categories, in particular the velocipedes, which consist of both bicycles and tricycles, are not high. Indeed, fine-grained correspondence on these highly dissimilar shapes remains a challenging problem.

Reliance on symmetry relations. In Table 3, we also report the performance of our algorithm when the input shapes do not come with detected symmetries. We simulate this case by applying our correspondence method over the ground truth set while ignoring all symmetry grouping. This test is meant to assess the dependence of our method on accurate symmetry detection. As can be seen, the precision and recall rates do degrade when symmetry is turned off. However, the drop is not as significant as one might have expected. 


\begin{tabular}{lcccccc}
\hline \multirow{2}{*}{ Category } & \multicolumn{2}{c}{$[\mathrm{Xu} 2012]$} & \multicolumn{2}{c}{ Ours (no sym) } & \multicolumn{2}{c}{ Ours } \\
& $\mathrm{P}$ & $\mathrm{R}$ & $\mathrm{P}$ & $\mathrm{R}$ & $\mathrm{P}$ & $\mathrm{R}$ \\
\hline chair & 0.68 & 0.36 & 0.67 & 0.42 & $\mathbf{0 . 7 6}$ & $\mathbf{0 . 5 3}$ \\
table & 0.58 & 0.36 & 0.70 & 0.41 & $\mathbf{0 . 7 5}$ & $\mathbf{0 . 4 4}$ \\
bed & 0.53 & 0.33 & 0.63 & 0.29 & $\mathbf{0 . 6 9}$ & $\mathbf{0 . 3 4}$ \\
airplane & 0.65 & 0.41 & 0.71 & 0.47 & $\mathbf{0 . 7 2}$ & $\mathbf{0 . 4 7}$ \\
velocipede & 0.45 & 0.25 & 0.49 & 0.35 & $\mathbf{0 . 5 1}$ & $\mathbf{0 . 3 7}$ \\
\hline
\end{tabular}

Table 3: Comparison on per-category precision $(P)$ and recall $(R)$ rates between our method and a baseline method [Xu et al. 2012] for matching shape pairs. Also shown (in the middle two columns under "no sym") are $P+R$ rates for our correspondence results which are obtained without having symmetry relations identified on the input shapes. Best results are shown in bold.

Comparison to Tevs et al. More comparable to our work in terms of input and objective is the symmetry-driven graph matching scheme of Tevs et al. [2014], which operates on a pair of shapes and returns correspondences between part ensembles with possibly different topologies. However, their search is not over topologychanging deformations, but through graph matchings over symmetry groups. We obtained two sets of models (bed and bicycle) from the authors of this work to make a comparison. They tested their algorithm on these models with disjoint parts and detected symmetries that our algorithm is able to take as input.

Figure 16 shows one set of matching results for each dataset obtained using our algorithm. With the deformation energy accounting for geometric distortion, our method is able to find the proper alignments between the beds and the bench. Matching between fine-level parts also appears to be more accurate. With a finegrained matching, topology blending is possible; see Figure 12.

\section{Discussion, limitations, and future work}

We present a deformation-driven method to produce a fine-grained correspondence between two 3D shapes that may differ in their geometry and topology substantially. The key challenge lies in how to obtain a fine-grained, topology-varying matching. We believe that a continuous, or rather, a piecewise continuous formulation is suitable for such a task. We solve the problem by following a deformation-driven paradigm, which was previously adopted to match curves and surfaces without allowing for topological variations. The main novelty of our work is the development of a deformation model, the GeoTopo transform, and an associated deformation energy that simultaneously accounts for geometric, structural, and topological shape variations. With the shape parts to be matched undergoing topological changes, such as part splits or duplications, the final matching is piecewise continuous.

Initial segmentation. The foremost limitation to our current work that one may readily point out is that we assume the two input shapes are "segmented into meaningful parts in a symmetry-aware manner." Such segmentations may not always be easy to obtain, especially for detailed 3D shapes found in online repositories. In Figure 17, we show automatic segmentation results computed by different state-of-the-art segmentation methods [Shapira et al. 2008; Au et al. 2012; Kaick et al. 2014] using their default parameters. It should be expected that using the same parameters for unsupervised segmentation methods would result in over- or under-estimation of part boundaries, producing imperfect segments.

When imperfect initial segmentations are provided, our method may fail to recover proper correspondences since a key criterion in our method is structure preservation while imperfect segmentations may not reflect the correct structures in the input shapes. The top

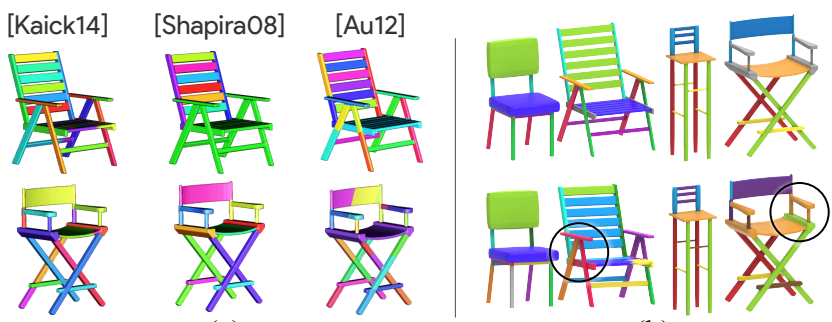

(a)

(b)

Figure 17: Initial segmentations by state-of-the-art automatic methods and the resulting correspondence. (a) Segmentations from three such methods using their default parameters. (b) Correspondence results using our method applied to original components with manual segmentation (top) vs. results initialized by automatic segmentations using the method of [Kaick et al. 2014] (bottom). In both cases we use the same original segmentation for the chair on the left. Clearly invalid correspondences are highlighted.

row of Figure 18 shows an example of corresponding shape pairs that are segmented in a symmetry-aware manner but have different segmentation granularity. As can be seen, the feet of the coarselysegmented robot are not correctly matched with the more finely segmented one. A similar issue can be seen in the chair model when the armrest is attached to the leg in the chair model on the left but not so in the one on the right.

Having said the above, even if perfect initial segmentations were available, matching two shapes characterized by these segmentations under significant geometric and topological variations and producing a fine-grained and piecewise-continuous matching remains a highly challenging task. With extensive evaluation, we have demonstrated the significant advance made by our work over the state-of-the-art methods, including those designed for coanalysis, when the compared methods were provided with exactly the same inputs and initial segmentations.

Furthermore, we believe that the utility of our deformation-driven analysis framework goes beyond correspondence search; it may also be applicable to actually finding the proper segmentations, or co-segmentations, of two shapes. The basic premise is that for two shapes to be matched in the most meaningful way, the matching ought to be on two meaningful segmentations. We leave the ensuing investigation for future work, providing here a preliminary verification. In Figure 19, we show the best correspondence our method finds based on four different segmentations. As can be observed, meaningful segmentations, even when they differ in part counts, result in similar deformation energy. In contrast, poor segmentations cannot be compensated for by a meaningful correspondence.

Dense correspondence. In this work, we have not evaluated our correspondence scheme using the benchmark of Kim et al. [2011]. The reasons are two-fold. First, the benchmark evaluation is based on measuring geodesic distortions, which would be inappropriate to judge topology-varying shape correspondences; such correspondence results are at best piecewise continuous. Second, our correspondence search is focused on curve-sheet structures and not their corresponding surfaces. Our method is not designed to compete with those aimed at near-isometric matching since we do not optimize for any criterion based on surface metrics.

Partial matching. Although one-to-many correspondences can be identified, our method is not designed to perform general partial matching, such as matching a hand model to a whole human shape. Such cases would entail a preponderance of outlier parts on the target side, largely increasing the difficulty of the combinato- 

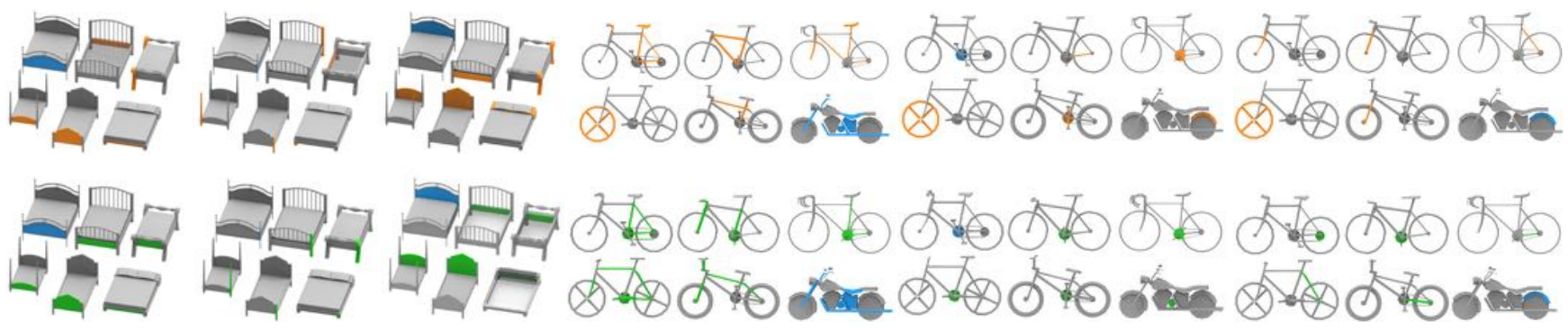

Figure 16: Comparison to Tevs et al. [2014]. Top row shows their results on the "bed" and "bicycle" sets. As shown in their paper, blue parts are the parts to be matched. Bottom row shows the correspondence for the same highlighted part using our method. Note that while the input is not assumed to be prealigned (as in the bench model), our method correctly identifies correspondences that are spatially accurate.
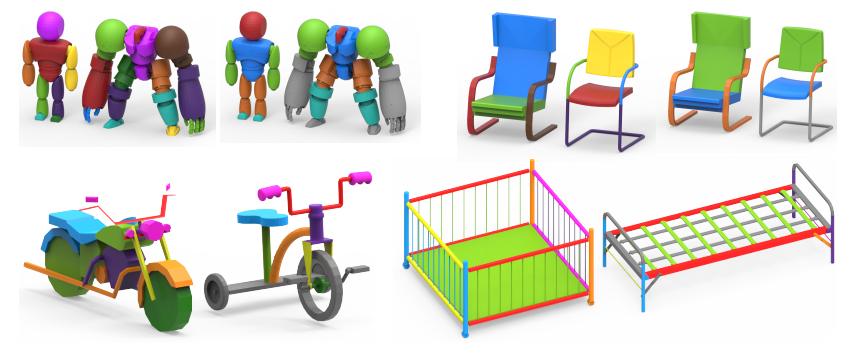

Figure 18: Several failure cases due to imperfect segmentation (top row) and large geometric deviation between parts that ought to be matched up (bottom row). On the top row, the initial segmentations for each pair of shapes are shown on the left. The bottom row shows the pairs having the worst ground-truth correspondence score in their respective sets. Our method does not consider partial matching, which would be the ideal solution for such pairs.

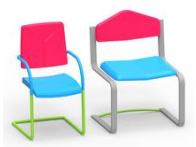

0.16

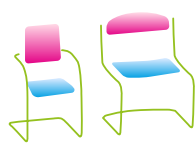

0.05

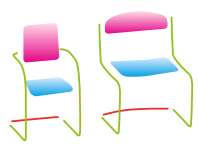

0.04

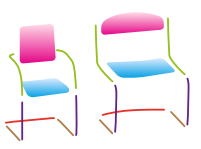

0.06
Figure 19: Segmentation and correspondence. Low quality correspondence result on the original input shapes (left). We apply different segmentations on the part abstractions and report the total correspondence cost. Note that while each segmentation has a different number of parts, the compatibility of these segments consistently results in low energy.

rial search. Also, defining a proper deformation energy for partial matching remains open; we leave this for future work.

Other limitations. First, like Alhashim et al. [2014], we focus on processing man-made shapes that can be well abstracted by curvesheet structures. If this assumption holds, our method produces reasonable results. However, there are limits to the degree with which these structures can represent broader categories of models. For example, buildings, cars, and other models where fine-scale details are important may not be adequately modeled by such abstractions. Furthermore, some models may include "tubes" (parts with a closed sheet skeleton) or parts made out of open surfaces for which there is no good skeleton. Such models would need to first be adequately abstracted before our method can be applied. Second, our method is purely geometric. There are cases where, even when the energy is very low for some correspondence, there is no semantic relationship between the matched parts. The bottom row of Figure 18 shows a few failure cases related to these issues.
Future work. Aside from addressing the limitations mentioned above, we would like to improve the quality of the in-between shape deformations generated during correspondence search. Such an improvement may allow us to more-efficiently explore topology variations in a large shape collection, as in the work of Ovsjanikov which operates on box proxies [Ovsjanikov et al. 2011]. Lastly, we would like to investigate two intriguing applications of our approach: deformation-driven shape segmentation and transitive shape correspondence, as discussed above.

\section{Acknowledgments}

We would like to thank the reviewers for their valuable comments and feedback. We also thank Vladimir Kim and Youyi Zheng for making available the source code of their methods. This work is supported in part by grants from NSERC Canada (611770) and NSFC China (61202333, 61363048, 61572507).

\section{References}

Aiger, D., Mitra, N. J., AND Cohen-Or, D. 2008. 4-points congruent sets for robust surface registration. ACM Trans. on Graphics 27, 3, 1-10.

Alhashim, I., Li, H., Xu, K., CaO, J., Ma, R., And Zhang, H. 2014. Topology-varying $3 \mathrm{D}$ shape creation via structural blending. ACM Trans. on Graphics 33, 4, Article 158.

Au, O. K.-C., COHEn-Or, D., TAI, C.-L., Fu, H., AND Zheng, Y. 2010. Electors voting for fast automatic shape correspondence. Computer Graphics Forum (Proc. EUROGRAPHICS) 29, 2.

Au, O.-C., Zheng, Y., Chen, M., Xu, P., And TaI, C.-L. 2012. Mesh segmentation with concavity-aware fields. Visualization and Computer Graphics, IEEE Transactions on 18, 7 (July), 1125-1134.

Blanz, V., AND Vetter, T. 1999. A morphable model for the synthesis of $3 \mathrm{~d}$ faces. In Proceedings of the 26th Annual Conference on Computer Graphics and Interactive Techniques, ACM Press/Addison-Wesley Publishing Co., New York, NY, USA, SIGGRAPH '99, 187-194.

BoKeloh, M., Wand, M., Koltun, V., And Seidel, H.-P. 2011. Pattern-aware shape deformation using sliding dockers. ACM Trans. on Graphics 30, 123:1-123:10.

Botsch, M., AND SoRKINE, O. 2008. On linear variational surface deformation methods. IEEE Trans. on Visualization and Computer Graphics (TVCG) 14, 1, 213-230. 
Gal, R., Sorkine, O., Mitra, N., And Cohen-Or, D. 2009. iWires: An Analyze-and-Edit Approach to Shape Manipulation. ACM Trans. on Graphics 28, 3, 33:1-33:10.

Gelfand, N., Mitra, N. J., Guibas, L. J., And Pottmann, H. 2005. Robust global registration. In Proc. Symp. on Geom. Processing (SGP), 197-206.

GolovinskiY, A., AND Funkhouser, T. 2009. Consistent segmentation of 3D models. Computers \& Graphics (Proc. SMI) $33,3,262-269$.

Hilaga, M., Shinagawa, Y., Kohmura, T., And Kunit, T. L. 2001. Topology matching for fully automatic similarity estimation of 3D shapes. In Proc. SIGGRAPH, 203-212.

Hu, R., FAn, L., AND LiU, L. 2012. Co-segmentation of 3D shapes via subspace clustering. Computer Graphics Forum (Proc. SGP) 31, 5, 1703-1713.

HuAnG, Q., AND Guibas, L. 2013. Consistent shape maps via semidefinite programming. Computer Graphics Forum (Proc. $S G P) 32,5$.

Huang, Q.-X., Adams, B., Wicke, M., And Guibas, L. J. 2008. Non-rigid registration under isometric deformations. Computer Graphics Forum (Proc. SGP) 27, 5, 1449-1457.

Huang, Q., Koltun, V., And Guibas, L. 2011. Joint shape segmentation with linear programming. ACM Trans. Graph. 30, 6 (Dec.), 125:1-125:12.

Huang, Q.-X., Su, H., and Guibas, L. 2013. Fine-grained semi-supervised labeling of large shape collections. ACM Trans. on Graphics 32, 6, 190:1-190:10.

Kaick, O. V., Fish, N., Kleiman, Y., Asafi, S., And CohenOR, D. 2014. Shape segmentation by approximate convexity analysis. ACM Trans. Graph. 34, 1 (Dec.), 4:1-4:11.

Kim, V. G., Lipman, Y., And Funkhouser, T. 2011. Blended intrinsic maps. ACM Trans. on Graphics 30, 4, 79:1-79:12.

Kim, V. G., Li, W., Mitra, N. J., Chaudhuri, S., DiVerdi, S., AND Funkhouser, T. 2013. Learning Part-based Templates from Large Collections of 3D Shapes. ACM Trans. on Graphics (Proc. SIGGRAPH) 32, 4.

Laga, H., Mortara, M., and Spagnuolo, M. 2013. Geometry and context for semantic correspondences and functionality recognition in man-made $3 \mathrm{~d}$ shapes. ACM Trans. on Graphics $32,5,150: 1-150: 16$.

Lin, J., Cohen-Or, D., Zhang, H. R., Liang, C., Sharf, A., Deussen, O., AND CHEN, B. 2011. Structure-preserving retargeting of irregular $3 \mathrm{~d}$ architecture. ACM Trans. on Graphics $30,6,183: 1-183: 10$.

Lipman, Y., Chen, X., Daubechies, I., And Funkhouser, T. 2010. Symmetry factored embedding and distance. $A C M$ Trans. on Graphics 29, 4, 103:1-12.

LOWERRE, B. T. 1976. The harpy speech recognition system. $\mathrm{PhD}$ thesis, Carnegie Mellon University.

Meng, M., Xia, J., Luo, J., And He, Y. 2013. Unsupervised co-segmentation for $3 \mathrm{~d}$ shapes using iterative multi-label optimization. Comput. Aided Des. 45, 2 (Feb.), 312-320.

Mitra, N., Wand, M., Zhang, H. R., Cohen-Or, D., Kim, V., AND HuANG, Q.-X. 2013. Structure-aware shape processing. In SIGGRAPH Asia 2013 Courses, 1:1-1:20.
Ovsjanikov, M., Li, W., Guibas, L., And Mitra, N. J. 2011. Exploration of continuous variability in collections of $3 \mathrm{~d}$ shapes. ACM Trans. on Graphics 30, 4, 33:1-33:10.

Russ, J. C. 2011. The Image Processing Handbook, Sixth Edition, 6th ed. CRC Press, Inc., Boca Raton, FL, USA.

Sederberg, T. W., And Greenwood, E. 1992. A physically based approach to 2-D shape blending. In Proc. SIGGRAPH, 25-34.

Shapira, L., Shamir, A., AND Cohen-Or, D. 2008. Consistent mesh partitioning and skeletonisation using the shape diameter function. Vis. Comput. 24, 4 (Mar.), 249-259.

Sidi, O., VAN KaICK, O., Kleiman, Y., Zhang, H., AND COHEN-OR, D. 2011. Unsupervised co-segmentation of a set of shapes via descriptor-space spectral clustering. ACM Trans. on Graphics 30, 6, 126:1-126:9.

Tevs, A., Huang, Q., Wand, M., Seidel, H.-P., And GUIBAS, L. 2014. Relating shapes via geometric symmetries and regularities. ACM Trans. on Graphics 33, 4, 119:1-119:12.

VAN Dongen, S. 2000. Graph Clustering by Flow Simulation. $\mathrm{PhD}$ thesis, University of Utrecht.

VAN Kaick, O., Zhang, H., Hamarneh, G., AND CohenOR, D. 2010. A survey on shape correspondence. Computer Graphics Forum 30, 6, 1681-1707.

van Kaick, O., Tagliasacchi, A., Sidi, O., Zhang, H., Cohen-Or, D., Wolf, L., And Hamarneh, G. 2011. Prior knowledge for part correspondence. Computer Graphics Forum (Proc. EUROGRAPHICS) 30, 2.

van Kaick, O., Xu, K., Zhang, H., Wang, Y., Sun, S., SHAMIR, A., AND COHEN-OR, D. 2013. Co-hierarchical analysis of shape structures. ACM Trans. on Graphics 32, 4, Article 69.

Wand, M., Adams, B., Ovsjanikov, M., Berner, A., BoKeloh, M., Jenke, P., Guibas, L., SeIdel, H.-P., AND SCHILling, A. 2009. Efficient reconstruction of non-rigid shape and motion from real-time 3D scanner data. ACM Trans. on Graphics 28, 2, 1-15.

Wang, Y., Xu, K., Li, J., Zhang, H., Shamir, A., LiU, L., Cheng, Z., AND Xiong, Y. 2011. Symmetry hierarchy of man-made objects. Computer Graphics Forum (Proc. EUROGRAPHICS) 30, 2, 287-296.

Xu, K., Li, H., Zhang, H., Cohen-Or, D., Xiong, Y., and CHENG, Z. 2010. Style-content separation by anisotropic part scales. ACM Trans. on Graphics 29, 5.

Xu, K., Zhang, H., Cohen-Or, D., And Chen, B. 2012. Fit and diverse: Set evolution for inspiring $3 \mathrm{~d}$ shape galleries. $A C M$ Trans. on Graphics (Proc. SIGGRAPH) 31, 4, 57:1-10.

Zhang, H., Sheffer, A., Cohen-Or, D., Zhou, Q., VAn KAICK, O., AND TAgliasaCCHI, A. 2008. Deformationdriven shape correspondence. Computer Graphics Forum (Proc. SGP) $27,5,1431-1439$.

Zheng, Y., Fu, H., Cohen-Or, D., Au, O. K.-C., And TAI, C.-L. 2011. Component-wise controllers for structurepreserving shape manipulation. Computer Graphics Forum (Proc. EUROGRAPHICS) 30, 2, 563-572.

Zheng, Y., Cohen-Or, D., Averkiou, M., And Mitra, N. J. 2014. Recurring part arrangements in shape collections. Computer Graphics Forum (Proc. EUROGRAPHICS) 33, 2. 